\title{
Musculoskeletal Modelling And Simulation Of Oil Palm Fresh Fruit Bunch Harvesting
}

\author{
Yon Sin Chan \\ Monash University Malaysia \\ Yu Xuan Teo \\ Monash University Malaysia \\ Darwin Gouwanda ( $\sim$ darwin.gouwanda@monash.edu ) \\ Monash University Malaysia \\ Surya Girinatha Nurzaman \\ Monash University Malaysia \\ Alpha Agape Gopalai \\ Monash University Malaysia \\ Subbiah Thannirmalai \\ Sime Darby Technology Centre
}

\section{Research Article}

Keywords:

Posted Date: March 7th, 2022

DOI: https://doi.org/10.21203/rs.3.rs-1414610/v1

License: (c) (i) This work is licensed under a Creative Commons Attribution 4.0 International License.

Read Full License 


\section{Abstract}

This study aims to create and evaluate an upper extremity musculoskeletal model of the oil palm harvesting motion and assess the Musculoskeletal Disorder (MSD) risk of this activity. Tests were conducted on six harvesters at a Malaysia oil palm plantation. Six Inertial Measurement Units (IMU) and Surface Electromyography (sEMG) were used to collect kinematics of the back, shoulder and elbow joints and measure the muscle activations of longissimus, multifidus, biceps and triceps. A musculoskeletal model was constructed and simulated using the IMU data. The results were benchmarked against the commercial motion capture tool and SEMG. It was found that joint angle had the maximum coefficient of correlation (R) of 0.99 and minimum Root Mean Square Error of $1.84^{\circ}$, whereas muscle activation had the maximum R of 0.87 and minimum Mean Absolute Error of 0.10 . This study showed that the longissimus was more actively used than multifidus, and the biceps was another primary muscle relied on during harvesting. The harvesters faced a higher risk of MSD while performing back flexion, back rotation, shoulder flexion and elbow flexion. Lastly, it demonstrated the feasibility of the musculoskeletal modelling and simulation to further investigate the upper extremity joints and muscles during harvesting.

\subsection{Introduction}

Investigating human motion is important in many fields with different purposes, such as improving workers' efficiency, posture and working conditions. Motion capture technologies, including the Motion Capture System (MoCap), Inertial Measurement Unit (IMU) and Electromyography (EMG), are commonly used to investigate the joint kinematics and muscle activations [1]. However, some important biomechanical parameters, such as the muscles forces, cannot be identified alone by these sensors. Hence, several musculoskeletal modelling and simulation tools were created and utilized to investigate human motion in various activities [2-5].

OpenSim (SimTK, Stanford, CA, USA) and Anybody Modeling System (Anybody, Aalborg, Denmark) are some of the popular tools used to perform musculoskeletal modelling and simulation in gait [2], running [3], lifting tasks [4] and bowling (cricket) [5]. Both software allow the user to create a musculoskeletal model, either building from scratch or modifying the existing model. The users can also perform relevant computation, e.g., kinematic analysis, inverse dynamics analysis and static optimization [6]. With the wider adoption of IMU in motion capture and analysis, the use of IMUs data in musculoskeletal modelling and simulation offers several additional advantages over the conventional method. IMU is small and light, easy to set up and can produce comparable results with MoCap [7]. It also allows the motion to be captured outside the laboratory environment. These attributes, in turn, produce a more realistic data that accurately represent the measured activity.

One of the fields where human motion analysis can be beneficial is agriculture. Palm oil has been the fastest-growing vegetable oil globally since 2004 [8]. One of the main activities during the upstream production of palm oil is Fresh Fruit Bunch (FFB) harvesting. FFB harvesting is performed manually by skilled harvesters, who visually identify and harvest the ripe FFB [9]. Several studies showed that the 
harvesters harvest the FFB in an awkward posture, that exposes them to a higher risk of Musculoskeletal Disorder (MSD) [10-14]. However, these studies use qualitative approaches to investigate this activity, such as questionnaires $[10,11]$, interviews $[10,12]$ and direct observation assessments, which include Rapid Entire Body Assessment (REBA) [13] and Rapid Upper Limb Assessment (RULA) [14]. A recent study [9] in 2021 investigated the FFB harvester's upper extremity motion using a quantitative approach. To the best of our knowledge, it is the first study investigating the FFB harvesting motion with IMU and EMG.

Although the existing studies sufficiently describe the dynamic behavior of the harvesters and the associated MSD risk, a computational model that can represent the harvesting motion has the potential to (1) elucidate some of the underlying biomechanical characteristics, (2) investigate the MSD risk associated with FFB harvesting and (3) serve as the fundamentals to devise strategies to improve the posture and mitigate or reduce the MSD risk. Therefore, this study aims to create an upper extremity musculoskeletal model that can represent the FFB harvesting motion and can be used to investigate the kinematics behavior of the back, shoulder and elbow, and their muscle activations. To validate this model, the joint angles of the back, shoulder and elbow were investigated and compared with results estimated by the commercial motion capture software. The muscle activations of the longissimus, multifidus, biceps and triceps were evaluated against the measurements collected using surface Electromyography (sEMG). Lastly, this study investigates the quality of the posture using the method proposed in [15] and its associated MSD risk.

\subsection{Methodology}

\subsection{MUSCULOSKELETAL MODEL AND SIMULATION}

A full-body musculoskeletal model created by Erica et al. [16] was modified to produce a model that is suitable for FFB harvesting activity. The original model has 30 body segments, 238 musculotendon actuators, 29 degrees of freedom and eight upper extremity Hill-type muscles, including the multifidus and longissimus muscles [16]. Three main modifications were made to the model as listed below:

1. The original Range of Motion (ROM) of shoulder and elbow joints were much greater than the Normative Range of Motion (NROM); hence they were modified to the NROM [17], as shown in Table 1. This modification prevents an awkward posture from being generated in OpenSim.

2. Hill-type biceps and triceps muscles were added. The muscle architectural parameters were adopted from an existing upper-extremity OpenSim model by Daniel et al. [18], used in pulling and pushing tasks. The Optical Muscle Fibre Length (OMFL), Tendon Slack Length (TSL) and Pennation Angle (PA) of the fascicles of biceps and triceps were reported as follows:

\OMFL: $11.6 \mathrm{~cm}$ (biceps long), $13.2 \mathrm{~cm}$ (biceps short), $13.4 \mathrm{~cm}$ (triceps long), $11.4 \mathrm{~cm}$ (triceps lateral), $11.4 \mathrm{~cm}$ (triceps medial)

\TSL: $27.8 \mathrm{~cm}$ (biceps long), $20.0 \mathrm{~cm}$ (biceps short), $14.3 \mathrm{~cm}$ (triceps long), $9.8 \mathrm{~cm}$ (triceps lateral), $9.1 \mathrm{~cm}$ (triceps medial) 
\PA: $0^{\circ}$ (biceps long), $0^{\circ}$ (biceps short), $12^{\circ}$ (triceps long), $9^{\circ}$ (triceps lateral), $9^{\circ}$ (triceps medial) [19]

3. Reserve actuators were added to the back joint (L5-S1), shoulder joints and elbow joints to provide extra actuation when the muscles cannot generate sufficient accelerations at a certain time. The optimal forces of the reserve actuators $\left(F_{R A}\right)$ must be low enough to ensure that the muscles are the main contributor to the net joint moments (i.e., the joint moment produced by the summed or net effect of all the structures) $[20,21]$. However, there is no recommended $F_{R A}$ for harvesting activity. Hence, five different values of $F_{R A}(10 N, 20 N, 30 N, 40 N$ and $50 N)$ were investigated and used to perform the inverse dynamics and calculate the net joint moments. Static optimization was performed to determine the reserve actuator moments. The peak reserve actuator moments at different $F_{R A}$ were normalized to the peak net joint moments. The results are presented in Table 2. The peak residual forces $\left(F_{X}, F_{Y}, F_{Z}\right)$ and peak residual moments $\left(M_{X}, M_{Y}, M_{Z}\right)$ were also added to the table. Since the normalized peak reserve actuator moments has to be less than $10 \%$ [22], only the $F_{R A}$ of $30 \mathrm{~N}, 40 \mathrm{~N}$ and $50 \mathrm{~N}$ were selected to estimate the muscle activation.

Table 1

ROM of the back, shoulder and elbow of the Erica et al.'s model and the proposed model (FE: FlexionExtension; LB: Lateral Bending; AA: Adduction-Abduction).

\begin{tabular}{|lllll|}
\hline Joint Motions & \multicolumn{2}{l}{$\begin{array}{l}\text { Erica } \text { et al.'s model [16] } \\
\text { Joint angle (Degree) }\end{array}$} & Maximum & \multicolumn{2}{l|}{$\begin{array}{l}\text { Minimum } \\
\text { Joint angle (Degree) }\end{array}$} & Maximum \\
\cline { 2 - 5 } & Minimum & 26 (Extension) & -80 (Flexion) & 26 (Extension) \\
\hline Back FE & -80 (Flexion) & 25 (Right) & -25 (Left) & 25 (Right) \\
\hline Back LB & -25 (Left) & 56 (Right) & -56 (Left) & 56 (Right) \\
\hline Back Rotation & -56 (Left) & 180 (Flexion) & -50 (Extension) & 180 (Flexion) \\
\hline Shoulder FE & -90 (Extension) & 90 (Adduction) & -180 (Abduction) & 0 (Adduction) \\
\hline Shoulder AA & -180 (Abduction) & (External & -80 (Internal & 60 (External \\
\hline $\begin{array}{l}\text { Shoulder } \\
\text { Rotation }\end{array}$ & -140 (Internal & 100 (Extation) & rotation) \\
\hline Elbow FE & -10 (Extension) & 160 (Flexion) & 0 (Extension) & 140 (Flexion) \\
\hline
\end{tabular}


Table 2

Average normalized peak reserve actuator moments of different joint motions $(n=6)$, peak residual forces and peak residual moments at five different $F_{R A}$.

\begin{tabular}{|c|c|c|c|c|c|}
\hline $\mathrm{F}_{\mathrm{RA}}$ & $10 \mathrm{~N}$ & $20 \mathrm{~N}$ & $30 \mathrm{~N}$ & $40 \mathrm{~N}$ & $50 \mathrm{~N}$ \\
\hline \multicolumn{6}{|c|}{ Average normalized peak reserve actuator moments of different joint motions (\%) } \\
\hline Back FE & 26.8287 & 13.3214 & 8.7991 & 6.6073 & 5.1785 \\
\hline Back LB & 8.4572 & 4.2350 & 2.8288 & 2.3165 & 1.7311 \\
\hline Back Rotation & 18.0534 & 8.9266 & 5.5598 & 5.2208 & 2.9981 \\
\hline Shoulder FE & 6.4938 & 3.3364 & 2.2446 & 1.4203 & 1.3648 \\
\hline Shoulder AA & 6.7106 & 3.6949 & 2.5009 & 3.2899 & 1.5137 \\
\hline Shoulder Rotation & 7.9474 & 4.0160 & 2.6183 & 2.3414 & 1.5426 \\
\hline Elbow FE & 9.0544 & 4.5270 & 3.0182 & 1.7002 & 1.8108 \\
\hline \multicolumn{6}{|c|}{ Peak residual forces $(\mathrm{N})$} \\
\hline $\mathrm{F}_{\mathrm{X}}$ & 134.4791 & 67.2394 & 44.8263 & 44.6750 & 26.8958 \\
\hline$F_{Y}$ & 127.4830 & 63.7415 & 42.4944 & 32.5968 & 25.4966 \\
\hline $\mathrm{F}_{\mathrm{Z}}$ & 144.0127 & 72.0064 & 48.0043 & 16.8280 & 28.8025 \\
\hline \multicolumn{6}{|c|}{ Peak residual moments $(\mathrm{Nm})$} \\
\hline$M_{x}$ & 112.6931 & 56.3466 & 37.5644 & 22.1164 & 22.5386 \\
\hline $\mathrm{M}_{\mathbf{Y}}$ & 29.0608 & 14.5304 & 9.6869 & 7.3175 & 5.8122 \\
\hline$M_{Z}$ & 100.4333 & 50.6165 & 34.0333 & 31.8223 & 20.4033 \\
\hline
\end{tabular}

Two assumptions were made in this study. The first assumption is that the exerted harvesting force of $303.5 \mathrm{~N}$ is the sum of the pulling force produced by the harvesters (300N [23]) and the weight of the harvesting tool (3.5N [24]). The second assumption is that the harvesting force was equally distributed to the left and right hands. Hence, an external force of $151.75 \mathrm{~N}$ was added to each hand.

Inverse kinematics was performed to calculate the joint angles. Static optimization was then used to estimate the muscle activations. It resolves the net joint moments into individual muscle forces at each instant while minimizing the sum of squared muscle activations [25]. It is the most conventional approach to calculate muscle activation during dynamic activities due to its robustness, higher efficiency and independence from the experimental EMG [26]. The simulated activation of all the muscles fascicles for each muscle were summed up [16]. For example, the estimated muscle activation of the longissimus is the total activations of the lumbar and thoracic components of the longissimus thoracis. The same applies to the multifidus, biceps and triceps. 


\subsection{EXPERIMENT}

The experiment was conducted at a Malaysia oil palm plantation. It involved six experienced right-handed male oil palm FFB harvesters $(33.5 \pm 6.0$ years, $168.83 \pm 4.74 \mathrm{~cm}, 56.83 \pm 4.26 \mathrm{~kg})$. They provided written consent after they were briefed on the experiment objectives and procedures. Informed written consent for publication was also provided. This study was reviewed and approved by Monash University Human Research Ethics Committee (MUHREC).

Six IMU sensors (APDM OPAL, Portland, OR, USA) were placed on the harvesters' sternum, lumbar, upper arms and wrists, as shown in Fig. 1(a). EMG electrodes (Biosignalplux, Lisbon, Portugal) were placed on the right side of the upper extremities to measure the muscle activations of the biceps, triceps, multifidus and longissimus with reference electrode at the $\mathrm{C} 7$ region (Fig. 1(b) and (c)). The locations of IMU sensors and EMG electrodes were based on the recommendations of APDM's software, Moveo Explorer (ME) [27] and SENIAM convention [28], respectively. Both sensors were synchronized using an external trigger. The sampling frequency of IMU and EMG sensors were $128 \mathrm{~Hz}$ and $1000 \mathrm{~Hz}$, respectively. The harvesters were requested to stand upright and remain stationary for three seconds for sensor calibration. They then performed the harvesting activity for one minute. The harvesters only harvested FFB from trees with a height between $3 \mathrm{~m}$ and $5 \mathrm{~m}$ for consistency purposes. A video camera was used to record the whole duration of the harvesting activity.

The data measured with IMU sensors were filtered with a 4th order Butterworth low-pass filter with a cutoff frequency of $6 \mathrm{~Hz}$ and were processed in two ways. The first method is denoted as IMU_ME. The raw IMU data were processed with ME (APDM, Portland, OR, USA), which used the Euler angle representation to calculate the joint angle [29]. The second method is named as IMU_OpenSim. The data were processed with OpenSim, which used the quaternion representation to calculate the joint angle [30]. The data measured with the EMG sensor were processed using code written in MATLAB (Mathworks, Nantucket, MA, USA). First, the data were filtered with a band-pass filter with $20 \mathrm{~Hz}$ and $450 \mathrm{~Hz}$ cut-off frequencies. They were then full-wave rectified and smoothened using a moving-average filter with a window size of $1000 \mathrm{~ms}$ [31]. The EMG data were then normalized by using the Peak Dynamic Method, which normalized the EMG data of each muscle to the maximum muscle activation of each harvester. This method is considered a reliable alternative to the conventional Maximum Voluntary Contraction (MVC) normalization method [32]. The MVC method was not used because of the remoteness of the plantation sites, which made it challenging to acquire the muscle MVC.

From the recorded video footage, it can be observed that the harvesting process is complex. The harvester needs to move around the tree, identify the ripe FFB and cut the fronds, which might interrupt the FFB harvesting. The harvester then positions the sickle on the FFB stalk and pull the sickle downward to cut the stalk, as shown in Fig. 2. For a more accurate and representative analysis, the IMU and EMG data for each harvesting motion of different time lengths were extracted, linearly interpolated and averaged to produce a harvesting motion with the same timeframe [33].

\subsection{MODEL VALIDATION}


The joint angles obtained with the IMU_OpenSim method were compared and validated with the IMU_ME method. The Pearson Correlation Coefficient (R)was calculated to determine the strength of association between these two methods. The closer the R to zero, the weaker the strength of association. Root Mean Square Error (RMSE) was also calculated to identify the error between them. A smaller RMSE value indicates a smaller difference between them. However, there is no clear guideline on the acceptable $\mathrm{R}$ and RMSE values. Hence, they were compared with the recent work by [34], which covers the range of R and RMSE that show good to excellent validity.

For comparison purposes, the EMG data were resampled to $128 \mathrm{~Hz}$ to match the sampling frequency of the IMU sensor. The muscle activations were normalized to the maximum muscle activation of each harvester. The simulated normalized muscle activations were then compared and validated with experimental data. The R and Mean Absolute Error (MAE) were calculated and compared with studies by $[35,36]$, which categorizes the range of $R$ and MAE into different qualities of correlation, shown in Table 3.

Table 3: Different qualities of correlation of the muscle activation for the range of $R$ and $\operatorname{MAE}[35,36]$.

\begin{tabular}{|l|llll|}
\hline \multicolumn{1}{|l}{$\mathbf{R}$} & Quality of correlation & MAE & Quality of correlation \\
\hline $0.9-1.0$ & Very high & $<0.10$ & Excellent \\
$0.7-0.9$ & High & $0.10-0.20$ & Good \\
\hline $0.5-0.7$ & Moderate & $>0.20$ & Poor \\
$0.3-0.5$ & Low & & \\
\hline $0.0-0.3$ & Negligible & & \\
\hline
\end{tabular}

To identify the active muscle used during harvesting, the normalized peak muscle activations during harvesting (at $F_{R A}=30 N, 40 N$ and $50 N$ ) were calculated and expressed in percentage. They were then ranked accordingly and compared with the ranking of the EMG results. The maximum ROM of each joint obtained with the IMU_OpenSim method were identified to determine the potential stressful joint motions during harvesting. The Discomfort Value (DV) proposed in [15] was used as a measure to identify the quality of the harvesting posture. It uses the ROM of the joint to determine the DV and then classifies it into different levels of perceived discomfort that correspond to the good, so-so, or poor posture. Different joint motions have different DV values. The relationship between NROM, DV and quality of postures for the back flexion is shown in Table 4. For example, if the ROM of the harvester's back flexion is $39^{\circ}$ and the NROM of this motion is $80^{\circ}$ [17], the harvester's back flexion will be equal to $48.75 \%$ of the NROM and will have a DV value of 22.08 , which is associated with a so-so posture. a higher DV value corresponds to a higher risk of MSD, and the joint may experience greater stress. These results were compared with the simulated muscle activations to confirm the dynamic behavior of the muscles during harvesting. 
Table 4

A relationship between the back flexion NROM, DV and quality of postures $[15,17]$.

\begin{tabular}{|lll|}
\hline NROM & DV & Quality of postures \\
\hline $0 \%$ & $<22.08$ & Good \\
\hline $25 \%-75 \%$ & $22.08-65.13$ & So-so \\
\hline $100 \%$ & $>65.13$ & Poor \\
\hline
\end{tabular}

\subsection{Result And Analysis}

\subsection{SIMULATION RESULTS}

A sequence of the simulated harvesting motion of a harvester is shown in Fig. 3. It can be observed that the following motions were performed during harvesting: back flexion, back lateral bending to the right, back rotation to the right, shoulder extension (left, right), shoulder adduction (left), shoulder internal rotation (left), shoulder abduction (right), shoulder external rotation (right), elbow flexion (left) and elbow extension (right). It took approximately 0.3 seconds to execute this movement. The extremely short duration of harvesting motion suggests that the harvesters used a very large force to cut the stalk and harvest the fruit. If this motion is performed repetitively, it may expose them to the potential risk of MSD.

Table 5 shows the average R and RMSE of the back, shoulder and elbow joint angles between the IMU_OpenSim and IMU_ME $\left(\mathrm{R}_{J A}, \mathrm{RMSE}_{J A}\right)$. These values correlate well with the good to excellent validity discussed in [34]. The $\mathrm{R}_{\mathrm{JA}}$ and $\mathrm{RMSE}_{\mathrm{JA}}$ of all the joint motions were well within the acceptable range. These results serve as solid evidence that the proposed musculoskeletal model can produce comparable results with the commercial IMU-based motion capture software. 
Table 5

The average R and RMSE of the back, shoulder and elbow joint angles between the IMU_OpenSim and IMU_ME $\left(R_{J A}, R M S E_{J A}\right)$ and the recent paper $\left(R_{J A}\right.$ in [34], $R_{M S E}$ in [34]).

\begin{tabular}{|c|c|c|c|c|}
\hline Types of motions & $\mathrm{R}_{\mathrm{JA}}$ & $R_{J A}$ in [34] & $\mathrm{RMSE}_{\mathrm{JA}}$ (degree) & $\mathrm{RMSE}_{\mathrm{JA}}$ in [34] (degree) \\
\hline \multicolumn{5}{|l|}{ Back } \\
\hline FE & 0.9821 & $0.7200-0.9900$ & 2.6405 & $1.8000-5.9000$ \\
\hline LB & 0.8469 & $0.7200-0.9900$ & 2.4630 & $1.8000-5.9000$ \\
\hline Rotation & 0.9453 & $0.7200-0.9900$ & 1.8418 & $1.8000-5.9000$ \\
\hline \multicolumn{5}{|c|}{ Shoulder (Dominant side) } \\
\hline FE & 0.9931 & $0.6900-1.0000$ & 9.9396 & $\leq 15.0000$ \\
\hline AA & 0.8836 & $0.6900-1.0000$ & 8.1467 & $<20.0000$ \\
\hline Rotation & 0.8965 & $0.6900-1.0000$ & 11.1098 & $1.0000-60.0000$ \\
\hline \multicolumn{5}{|c|}{ Shoulder (Non-dominant side) } \\
\hline $\mathrm{FE}$ & 0.9984 & $0.6900-1.0000$ & 11.5114 & $\leq 15.0000$ \\
\hline AA & 0.8127 & $0.6900-1.0000$ & 13.7612 & $<20.0000$ \\
\hline Rotation & 0.8379 & $0.6900-1.0000$ & 14.1523 & $1.0000-60.0000$ \\
\hline \multicolumn{5}{|c|}{ Elbow (Dominant side) } \\
\hline FE & 0.8582 & $0.8500-0.9900$ & 11.8733 & $0.2000-30.6000$ \\
\hline \multicolumn{5}{|c|}{ Elbow (Non-dominant side) } \\
\hline FE & 0.9959 & $0.8500-0.9900$ & 20.2215 & $0.2000-30.6000$ \\
\hline
\end{tabular}

Table 6 shows the average R and MAE of the muscle activations for the longissimus, multifidus, biceps and triceps between OpenSim and $E M G\left(R_{M A}, M A E_{M A}\right)$ with different $F_{R A}$. Longissimus and triceps showed high correlations of $R_{M A}$ and good correlations of $M A E_{M A}$ at all $F_{R A}$. Multifidus showed moderate $\left(F_{R A}=40 N\right)$ to high $\left(F_{R A}=30 N, 50 N\right)$ correlations of $R_{M A}$ and good correlation of $M A E_{M A}$ at all $F_{R A}$. Biceps showed moderate $\left(F_{R A}=40 N, 50 N\right)$ to high $\left(F_{R A}=30 N\right)$ correlations of $R_{M A}$ and poor correlation of $M A E_{M A}$ at all $F_{R A}$. Overall, the simulated activations of the longissimus and triceps showed good correlations with the EMG data at all $\mathrm{F}_{\mathrm{RA}}$. On the other hand, biceps showed the best correlation when the $F_{R A}$ was $30 N$, whereas multifidus showed the worst correlation when the $F_{R A}$ was $40 N$. 
Table 6

The average $R$ and MAE of the muscle activations for the longissimus, multifidus, biceps and triceps between OpenSim and EMG

$\left(R_{M A}, M A E_{M A}\right)$ at different values of $F_{R A}$.

\begin{tabular}{|llll|}
\hline Muscles & \multicolumn{3}{l}{$\mathrm{R}_{\mathrm{MA}}$ at different $\mathrm{F}_{\mathrm{RA}}$} \\
\cline { 2 - 4 } & $\mathbf{3 0 N}$ & $\mathbf{4 0 N}$ & $\mathbf{5 0 N}$ \\
\hline Longissimus & 0.8576 & 0.8407 & 0.8654 \\
\hline Multifidus & 0.8728 & 0.6669 & 0.8722 \\
\hline Biceps & 0.7225 & 0.6794 & 0.6568 \\
\hline Triceps & 0.7182 & 0.7700 & 0.7824 \\
\hline Muscles & MAE $_{\mathrm{MA}}$ at different & $\mathrm{F}_{\mathrm{RA}}$ \\
& $30 \mathrm{~N}$ & $\mathbf{4 0 N}$ & $\mathbf{5 0 N}$ \\
\hline Longissimus & 0.1045 & 0.1283 & 0.1019 \\
\hline Multifidus & 0.1306 & 0.1349 & 0.1359 \\
\hline Biceps & 0.2426 & 0.2406 & 0.2281 \\
\hline Triceps & 0.1252 & 0.1802 & 0.1829 \\
\hline
\end{tabular}

Generally, it is more difficult to obtain a good correlation in the muscle activation between simulation results and experimental data compared to joint angles. It is because many parameters may affect muscle activation in a simulated environment. These parameters include the estimated joint angle, harvesting force, muscle architectural parameter and reserve actuator. Moreover, the limitations of sEMG, such as movement artefacts, also might contribute to the discrepancies. On the other hand, only the limitations of IMU and the length of the body segment may affect the estimated joint angle. Hence, the recommended values of $\mathrm{R}$ to represent good validity are generally lower for muscle activation than for joint angle.

Figure 4 shows the average normalized peak muscle activation of the harvesters. Higher activation value indicated that the muscle was more active than the others during harvesting. With the musculoskeletal model, the ranking of the normalized peak muscle activations was the same when $\mathrm{F}_{\mathrm{RA}}$ were $30 \mathrm{~N}$ and $40 \mathrm{~N}$, as follows: longissimus $(60.57 \%, 60.35 \%)$, biceps $(55.79 \%, 53.55 \%)$, multifidus $(53.14 \%, 52.28 \%)$ and triceps $(50.66 \%, 44.55 \%)$. When $\mathrm{F}_{\mathrm{RA}}$ was $50 \mathrm{~N}$, longissimus $(62.37 \%)$ ranked first, followed by multifidus (56.58\%), biceps $(54.89 \%)$ and triceps (53.93\%). These rankings were different from the results obtained with EMG. Biceps (57.71\%) was found to have the highest activation, followed by longissimus (57.42\%), triceps $(56.31 \%)$ and multifidus (44.88\%). The results showed that the longissimus was more active than 
multifidus when performing the back joint motions. The biceps was more active than the triceps during harvesting.

\subsection{HARVESTING POSTURE QUALITY AND POTENTIAL RISK OF MSD}

Table 7 shows the percentage of harvesters with poor, so-so and good postures for different types of back, shoulder and elbow joint motions, obtained with the IMU_OpenSim method. The muscles responsible for each joint motion were included. No poor posture was observed during harvesting. Some or all harvesters showed so-so postures at the back flexion (33.33\%), back rotation (100.00\%), dominant shoulder rotation (33.33\%), non-dominant shoulder flexion (66.67\%), non-dominant shoulder rotation (33.33\%) and both elbow flexions (100\%). The joint motions with so-so posture indicate the potential of stressful joint motions, suggesting the risk of MSD. 
Table 7

Percentage of harvesters with poor, so-so and good postures for different types of back, shoulder and elbow joint motions $(n=6)$, obtained with IMU_OpenSim method and their associated muscles [37].

$\begin{array}{lllll}\begin{array}{l}\text { Types of } \\ \text { motions }\end{array} & \text { Muscles } & \begin{array}{l}\text { Poor } \\ \text { posture }\end{array} & \begin{array}{l}\text { So-so } \\ \text { posture }\end{array} & \text { Good } \\ \text { posture }\end{array}$

Back

\begin{tabular}{lllll} 
Flexion & Rectus abdominis & $0.00 \%$ & $33.33 \%$ & $66.67 \%$ \\
\hline LB & Longissimus, multifidus & $0.00 \%$ & $0.00 \%$ & $100.00 \%$ \\
\hline Rotation & & $0.00 \%$ & $100.00 \%$ & $0.00 \%$
\end{tabular}

\section{Shoulder (Dominant side)}

\begin{tabular}{|lllll}
\hline Flexion & Biceps & $0.00 \%$ & $0.00 \%$ & $100.00 \%$ \\
\hline Adduction & Triceps & $0.00 \%$ & $0.00 \%$ & $100.00 \%$ \\
\hline Rotation & $\begin{array}{l}\text { Infraspinatus, teres minor, teres } \\
\text { major }\end{array}$ & $0.00 \%$ & $33.33 \%$ & $66.67 \%$ \\
\hline
\end{tabular}

Shoulder (Non-dominant side)

\begin{tabular}{|lllll}
\hline Flexion & Biceps & $0.00 \%$ & $66.67 \%$ & $33.33 \%$ \\
\hline Adduction & Triceps & $0.00 \%$ & $0.00 \%$ & $100.00 \%$ \\
\hline Rotation & $\begin{array}{l}\text { Infraspinatus, teres minor, teres } \\
\text { major }\end{array}$ & $0.00 \%$ & $33.33 \%$ & $66.67 \%$ \\
\hline
\end{tabular}

Elbow (Dominant side)

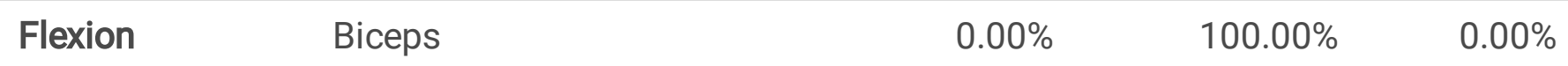

\section{Elbow (Non-dominant side)}

$\begin{array}{lllll}\text { Flexion } & \text { Biceps } & 0.00 \% & 100.00 \% & 0.00 \%\end{array}$

By comparing these joint motions with their corresponding muscles, it can be confirmed that the biceps and back muscles (longissimus and multifidus) were actively used during harvesting. The stressful joint motions with a higher risk of MSD were back flexion, back rotation, shoulder flexion and elbow flexion. This study did not measure the muscles responsible for back flexion (i.e., rectus abdominis) and shoulder rotation (i.e., infraspinatus, teres minor and teres major) [37]. Rectus abdominis is a superficial muscle, but it is covered by a thick layer of body fat, making it difficult to measure with sEMG [9]. The infraspinatus, teres minor and teres major are deep muscles [38], which cannot be measured with sEMG.

\section{Discussions}

This study created and simulated a musculoskeletal model that mimicked the harvesting motion. This model was adopted from a recent model by Erica et al. [16], which was the first validated lifting model in 
OpenSim. The lifting motion requires the model to frequently flex the trunk and lift the object. Hence, the back muscles of the Erica et al. model were ensured to be strong enough to perform the lifting motion. This advantage made it preferred for the harvesting model because many qualitative studies reported that the FFB harvesters complained of low back pain $[10,11,14]$, indicating that strong back muscles were required during harvesting. Moreover, many current OpenSim models assume the trunk as one rigid body, such as the full-body models by Rajagopal et al. [39] and Hamner et al. [40], which is not true in reality and may not fully represent the dynamic behavior of the trunk. The trunk in the Erica et al. model was made of several rigid body segments, including each lumbar vertebrae (L1 - L5), pelvis and torso [16]. This trunk structure is considered more accurate because the trunk of a real human is a chain of interconnected vertebrae, pelvis and scapula [21]. On the other hand, the biceps and triceps of the simulated harvester were added from another latest validated model by Daniel et al. [18], which performed pushing and pulling tasks at different elevation angles. The arm muscles were added from this model because the harvesting motion involves pulling motion, similar to the pulling task.

This study demonstrated a novel approach to investigate the worker's behavior in harvesting oil palm FFB. Wearable IMU allows the motion to be captured in the oil palm plantation without sophisticated setup and calibration. It is also proven to be cheaper, lighter and does not suffer from marker occlusion, making it an ideal alternative for outdoor applications than MoCap [41]. Two different representations and platforms were used to determine the upper extremity joint angles during harvesting. The IMU_ME method uses the Euler angle representation to calculate the joint angles [29], whereas the IMU_OpenSim method uses the quaternion representation to determine the joint angles [30]. Despite their differences, the results showed that the outcomes of the IMU_OpenSim method highly resemble the one derived using the IMU_ME method. The $R_{J A}$ and $R_{M S E_{J A}}$ were within the good to excellent range proposed in [34]. This suggests that OpenSim can be a valid tool to determine the kinematic behavior of the FFB harvester's upper extremity.

Although not directly comparable, the $\mathrm{R}_{\mathrm{JA}}$ and $\mathrm{RMSE}_{\mathrm{JA}}$ reported in this study were very close to those reported in other studies [42, 43], which calculate and validate their joint motions with different sensors. In a study for the upper extremity motions, Rodriogo Perez et al. investigated the joint motions when the subjects performed simple motions involving shoulder joint, elbow joint and wrist joint. The joint motions were measured with IMU sensors, validated with MoCap. The shoulder AA $(R=0.718)$ showed the lowest correlation when compared to shoulder FE $(R=0.994)$ and shoulder rotation $(R=0.995)$ [42]. This finding is consistent with the current study, suggesting that it is difficult to obtain a similar result for shoulder AA, even for simple motions. The authors then investigated the motions when the subjects served water from a jar. In this case, the shoulder rotation $(R=0.853)$ showed the lowest correlation when compared to shoulder FE $(R=0.996)$ and shoulder AA $(R=0.908)$ [42]. Jim Richards also pointed out that many papers have focused on sagittal plane joint angles rather than the coronal and transverse planes [31]. These previous findings support our findings, which show that shoulder FE has the highest correlation compared to other shoulder motions. 
In another study, Brice Bouvier et al. investigated the joint motions when the subjects performed simple elbow FE motions. The joint motions were measured with IMU sensors, validated with MoCap and has an RMSE value of $24^{\circ}$ [43]. A recent systematic review has shown that the validity of joint motions decreases when the level of complexity of the motions increases [34]. This implies that if the $R_{J A}$ and $R M S E_{J A}$ in this study are similar or better than those studies with simple motions, it can be assumed that the findings are acceptable. Since the RMSE $\mathrm{JA}_{\mathrm{A}}$ of elbow FE reported in this study (Dominant: $\mathrm{RMSE}_{\mathrm{JA}}$ $=11.8733^{\circ}$; Non-dominant: $\mathrm{RMSE}_{\mathrm{JA}}=20.2215^{\circ}$ ) were less than the RMSE obtained in the simple motion study $\left(\mathrm{RMSE}=24.0000^{\circ}\right)$, therefore the results are acceptable.

This study has also provided a novel approach to investigate the muscle activation of the oil palm FFB harvesting motion using OpenSim. The simulated normalized muscle activations were validated with normalized sEMG, which is the conventional standard used in human motion analysis [26, 44]. Other validation methods such as comparison with similar studies are impossible because very limited studies investigated the muscle behavior of FFB harvesters with EMG [9]. From Table 6, the normalized activations of the back muscles (longissimus and multifidus) showed a high correlation in $\mathrm{R}_{\mathrm{MA}}$ and a good correlation in $M A E_{M A}$ when $F_{R A}$ were $30 \mathrm{~N}$ and $50 \mathrm{~N}$. These findings concur with previous studies in lifting tasks $[16,45]$ and daily living activities [45], which obtained high similarity results for the back muscles between the OpenSim muscle activations and EMG.

For the arm muscles, the triceps demonstrated a high correlation in $R_{M A}$ and a good correlation in $M A E_{M A}$. The biceps demonstrated a high correlation in $R_{M A}$ and a poor correlation in $M A E_{M A}$ when $F_{R A}$ was $30 N$. Three possible reasons may explain the poor correlation of biceps. Firstly, higher speed can result in higher deviations than lower speed [46]. The harvesters must use a large force at a very high speed to harvest the fruit [47]. Since the biceps is the primary muscle to perform this motion $[9,48]$, it is reasonable that the biceps showed higher $\mathrm{MAE}_{\mathrm{MA}}$ values when compared to other muscles. Secondly, Roberto Bortoletto et al. proved that different $\mathrm{F}_{\mathrm{RA}}$ could affect the simulated muscle activation in OpenSim [49]. From Table 6, it can be observed that the multifidus and longissimus showed the best correlation of $R_{M A}$ and $M A E_{M A}$ when the $F_{R A}$ were $30 \mathrm{~N}$ and $50 \mathrm{~N}$, respectively. The biceps showed the highest $R_{M A}$ when $F_{R A}$ was $30 \mathrm{~N}$, whereas it showed the lowest $M A E_{M A}$ when $F_{R A}$ was $50 \mathrm{~N}$. The triceps showed the highest $R_{M A}$ when $F_{R A}$ was $50 \mathrm{~N}$, whereas it showed the lowest $M A E_{M A}$ when $F_{R A}$ was $30 N$. These findings are valuable and important because currently there aren't any recommended value of $F_{R A}$ for harvesting activity in OpenSim. Thirdly, the muscle architectural parameters of the simulated harvester may not represent the muscle architectural parameters of the real harvesters.

The potential risk of MSD that harvesters face during oil palm FFB harvesting were discussed based on the joint angles and muscle activations obtained with OpenSim. It was found that the back flexion, back rotation, shoulder flexion and elbow flexion were stressful joint motions during harvesting. During harvesting, the back muscles (longissimus and multifidus) and biceps were actively used. These findings are consistent with many qualitative studies on harvesters. Through questionnaires and direct interviews, many harvesters reported discomforts on their lower back $[10,11,14,50]$. Many studies that use the 
direct observation assessment method also found out that the harvesters were facing a prevalence of MSD on their backs $[14,48,50]$, shoulders (upper arms) $[47,50]$ and elbows (lower arms) $[14,47,48,50]$.

In a study by M. Faiz Syuaib [48], he analyzed the harvesting motions based on a video. He found out that the flexions of the back, shoulder and elbow were always stretched to the NROM of the joints. This result agrees with the findings reported in this study. However, the author did not analyze the rotation of the back joint. This may be due to the limitation of his approach because it is very difficult to measure the joint angle of rotation based on a video. In a recent study by $Y X$ Teo et al. [9], the authors used the sEMG and IMU to investigate the joint motions and muscles used during harvesting activity. They found that the longissimus, multifidus and biceps were the main muscles, consistent with this study's findings. They also reported that the back extension, back LB, shoulder flexion, elbow supination and elbow flexion were at risk of MSD. Other than the flexions of the shoulder and elbow, the other joint motions were not consistent with the findings in this study. This result may be due to the different focus of the studies. The previous study analyzed longer harvesting motions, which lasted for a few seconds. On the other hand, this study only analyzed the harvesting motion at that instant the fruit was harvested, which lasted approximately 0.3 seconds. It is motivated by the indication that this particular harvesting motion could be the main contribution of the MSD for the harvesters [13].

It was observed that many qualitative studies reported the harvesters suffered low back pain. A few possible reasons can explain this issue. First, it was found that after a long duration of repeated back flexion, the stiffness of the spine decreased. The deformation of the intervertebral disc and the stretching of the ligaments in the spine generated a change in the loading pattern, leading to low back pain [51]. Second, during the flexions of the back and shoulder, the moment arms of the body segments increase, causing the joint moment and compensatory tension in the back muscles to increase. It is due to the extremely small moment arms of the back muscles; hence a large force is required to counteract the joint moment [51]. Lastly, it was known that the passive moment (i.e., moment due to passive structures such as ligaments, fascia and cartilage) increases exponentially when a joint is approaching the ROM of flexion [21]. A large increase of passive moment might also lead to low back pain in the harvesters. All these evidence supported our findings, which proved that the flexions of the back joint, shoulder joints and elbow joints were the stressful joint motions during harvesting.

There are some limitations to this study. The muscle architectural parameters of the musculoskeletal model, such as the force-length relationship of muscle and tendon, may not represent the muscle architectural parameters of the real harvesters $[26,35,46]$. Moreover, the assumption that the harvesting force was $303.5 \mathrm{~N}$ may not represent the actual harvesting force for each harvester. The harvesting force is also influenced by factors such as the frond maturity, cutting angle, speed of cutting and the sharpness of the cutting edge of the harvesting tools [52]. It is suggested that a mock harvesting environment should be prepared indoors. A more controlled indoor environment will allow the other measurements such as MoCap and force sensor to improve the accuracy of the results. It has to bear in mind that although all these limitations of modelling software limit the extent of interpretation, they do not invalidate the model results [21]. The discrepancies between OpenSim and sEMG might be caused by the 
limitation of sEMG. There might be crosstalk and movement artefacts from neighbouring muscles [53, 54], especially during fast motions [26]. The small recording region of the sEMG electrodes on a particular muscle may also not represent the whole muscle's activation [26]. The fine-wire EMG would be a more direct and accurate approach to validate OpenSim results. However, it is invasive and is not practical for field study [26].

\section{Conclusion}

This study created and evaluated an upper extremity musculoskeletal model for FFB harvesting. The joint angles were validated against the commercial IMU-based motion capture software, whereas the muscle activations were benchmarked against the EMG data. The findings concur with previous qualitative studies that suggested the harvesters are exposed to the ergonomic risk of MSD, particularly the back flexion, back rotation, shoulder flexion and elbow flexion. The muscles actively used during harvesting were the longissimus, multifidus and biceps. This study showed that the musculoskeletal modelling and simulation tool such as OpenSim can be a useful method to investigate the biomechanical behavior of the harvester during harvesting and deriving some important parameters that are difficult to be measured directly, such as joint moments, muscle forces and activation of the deep muscles. Furthermore, these findings can provide essential information for future studies that focus on the ergonomics of the harvesting activity and the design and development of a wearable device to assist the harvester.

\section{Declarations}

\section{AUTHOR CONTRIBUTIONS}

YSC: Writing, Data Collection, Methodology, Data Processing

YXT: Methodology, Data Collection

DG: Methodology, Writing Reviewing and Editing, Supervision

SGN: Methodology, Writing Reviewing, Supervision

AAG: Methodology, Writing Reviewing, Supervision

S.T.: Supervision, Data Collection

\section{COMPETING INTERESTS}

The authors declare no competing interests.

\section{ADDITIONAL INFORMATION}

Yon Sin Chan and Yu Xuan Teo are supported by Graduate Research Merit Scholarship, Monash University Malaysia. This study was also supported by Advanced Engineering Platform and Monash 
Industry Palm Oil Research Platform, Monash University Malaysia, as well as project entitled "Analysis of Fresh Fruit Bunch Cutters' and Loose Fruit Collectors' Dynamics: Towards soft wearable robots to increase their productivity" from Sime Darby.

\section{References}

1. Castillo-Escario, Y., et al., Assessment of trunk flexion in arm reaching tasks with electromyography and smartphone accelerometry in healthy human subjects. Scientific reports, 2021. 11(1): p. 1-13.

2. Damsgaard, M., et al., Analysis of musculoskeletal systems in the AnyBody Modeling System. Simulation Modelling Practice and Theory, 2006. 14(8): p. 1100-1111.

3. Nitschke, M., et al., Efficient trajectory optimization for curved running using a 3D musculoskeletal model with implicit dynamics. Scientific reports, 2020. 10(1): p. 1-12.

4. Behjati, M. and N. Arjmand, Biomechanical assessment of the NIOSH lifting equation in asymmetric load-handling activities using a detailed musculoskeletal model. Human factors, 2019. 61(2): p. 191-202.

5. Wells, D., et al., Prescribing joint co-ordinates during model preparation to improve inverse kinematic estimates of elbow joint angles. Journal of biomechanics, 2017. 51: p. 111-117.

6. Seth, A., et al., OpenSim: Simulating musculoskeletal dynamics and neuromuscular control to study human and animal movement. PLoS computational biology, 2018. 14(7): p. e1006223.

7. Gouwanda, D. and S. Senanayake. Emerging trends of body-mounted sensors in sports and human gait analysis. in 4th Kuala Lumpur International Conference on Biomedical Engineering 2008. 2008. Springer.

8. A.H.Ling, Global palm oil trade-prospects and outlook. 2019.

9. Teo, Y.X., et al., Quantification of muscles activations and joints range of motions during oil palm fresh fruit bunch harvesting and loose fruit collection. Scientific Reports, 2021. 11(1): p. 1-13.

10. Tewtow, S., P. Bhuanantanondh, and K. Mekhora, Prevalence of Work-Related Musculoskeletal Disorders among Thai Oil Palm Workers in Khao Phanom District, Krabi Province, Thailand. Industrial Engineering \& Management Systems, 2019. 18(4): p. 630-637.

11. Bhuanantanondh, P., et al., The Prevalence of and Risk Factors Associated with Musculoskeletal Disorders in Thai Oil Palm Harvesting Workers: A Cross-Sectional Study. International Journal of Environmental Research and Public Health, 2021. 18(10): p. 5474.

12. Ng, Y.G., et al., Risk factors of musculoskeletal disorders among oil palm fruit harvesters during early harvesting stage. Annals of Agricultural and Environmental Medicine, 2015. 22(2).

13. Nawi, N.S.M., B. Md Deros, and N. Norani. Assessment of oil palm fresh fruit bunches harvesters working postures using REBA. in Advanced Engineering Forum. 2013. Trans Tech Publ.

14. Deros, B., et al. Investigation of oil palm harvesters' postures using RULA analysis. in 2014 IEEE conference on biomedical engineering and sciences (IECBES). 2014. IEEE. 
15. Kee, D. and W. Karwowski, Ranking systems for evaluation of joint and joint motion stressfulness based on perceived discomforts. Applied ergonomics, 2003. 34(2): p. 167-176.

16. Beaucage-Gauvreau, E., et al., Validation of an OpenSim full-body model with detailed lumbar spine for estimating lower lumbar spine loads during symmetric and asymmetric lifting tasks. Computer methods in biomechanics and biomedical engineering, 2019. 22(5): p. 451-464.

17. Norkin, C.C. and D.J. White, Measurement of joint motion: a guide to goniometry. 2016: FA Davis.

18. McFarland, D.C., et al., Spatial Dependency of Glenohumeral Joint Stability During Dynamic Unimanual and Bimanual Pushing and Pulling. Journal of biomechanical engineering, 2019. 141(5).

19. Saul, K.R., et al., Benchmarking of dynamic simulation predictions in two software platforms using an upper limb musculoskeletal model. Computer methods in biomechanics and biomedical engineering, 2015. 18(13): p. 1445-1458.

20. Hicks, J.L., et al., Is my model good enough? Best practices for verification and validation of musculoskeletal models and simulations of movement. Journal of biomechanical engineering, 2015. 137(2).

21. Robertson, D.G.E., et al., Research methods in biomechanics. 2013: Human kinetics.

22. Hicks, J. Reserves and Residuals. 27 June 2018; Available from: https://simtkconfluence.stanford.edu:8443/display/OpenSim33/_reserves+and+residuals

23. Ahmad, M.R., et al., THE EFFECT OF DESIGN PARAMETERS ON THE FORCE AND ENERGY REQUIREMENT FOR CUTTING OIL PALM FRONDS USING MAGNETIC FORCE. Jurnal Teknologi, 2020. 82(4).

24. Advantages of Palm King Set. 2021; Available from: https://www.klspalmking.com/index.php? ws=showproducts\&products_id=1408555\&cat=PK-Safety-Tool-Box-Set.

25. Hicks, J. How Static Optimization Works. 1 November 2021]; Available from: https://simtkconfluence.stanford.edu:8443/display/OpenSim/How+Static+Optimization+Works.

26. Trinler, U.K., Muscle force estimation in clinical gait analysis. 2016, University of Salford.

27. Burnett, I. Moveo Explorer - Strap and Opal Placement. 9 March 2019; Available from: https://support.apdm.com/hc/en-us/articles/360025078971-Moveo-Explorer-Strap-Opal-Placement.

28. Recommendations for sensor locations on individual muscles. 20 October 2021]; Available from: http://www.seniam.org/.

29. Holmstrom, L. Understanding joint angle estimation with Moveo Explorer. 13 October 2018; Available from: https://support.apdm.com/hc/en-us/articles/360015418912-Understanding-Joint-AngleEstimation-with-Moveo-Explorer

30. Holmstrom, L. How is the orientation of an Opal estimated. 1 May 2021; Available from: https://support.apdm.com/hc/en-us/articles/115000390803-How-is-the-orientation-of-an-Opalestimated-

31. Richards, J., The Comprehensive Textbook of Clinical Biomechanics. 2018: Elsevier Health Sciences. 
32. Ghazwan, A., et al., Can activities of daily living contribute to EMG normalization for gait analysis? PLoS One, 2017. 12(4): p. e0174670.

33. Ren, Y., et al. Smartphone based user verification leveraging gait recognition for mobile healthcare systems. in 2013 IEEE international conference on sensing, communications and networking (SECON). 2013. IEEE.

34. Poitras, I., et al., Validity and reliability of wearable sensors for joint angle estimation: A systematic review. Sensors, 2019. 19(7): p. 1555.

35. Karimi, M.T., et al., Determination of the correlation between muscle forces obtained from OpenSim and muscle activities obtained from electromyography in the elderly. Physical and Engineering Sciences in Medicine, 2021. 44(1): p. 243-251.

36. Odle, B., et al., Construction and evaluation of a model for wheelchair propulsion in an individual with tetraplegia. Medical \& biological engineering \& computing, 2019. 57(2): p. 519-532.

37. Muscolino, J.E., The muscular system manual-E-Book: the skeletal muscles of the human body. 2016: Elsevier Health Sciences.

38. Radhakrishnan, R. What Are Shoulder Muscles Called? 12 February 2021; Available from: https://www.medicinenet.com/what_are_shoulder_muscles_called/article.htm.

39. Rajagopal, A., et al., Full-body musculoskeletal model for muscle-driven simulation of human gait. IEEE transactions on biomedical engineering, 2016. 63(10): p. 2068-2079.

40. Hamner, S.R., A. Seth, and S.L. Delp, Muscle contributions to propulsion and support during running. Journal of biomechanics, 2010. 43(14): p. 2709-2716.

41. Müller, B., et al., Handbook of Human Motion. 2018: Springer Berlin.

42. Pérez, R., et al., Upper limb portable motion analysis system based on inertial technology for neurorehabilitation purposes. Sensors, 2010. 10(12): p. 10733-10751.

43. Bouvier, B., et al., Upper limb kinematics using inertial and magnetic sensors: Comparison of sensorto-segment calibrations. Sensors, 2015. 15(8): p. 18813-18833.

44. Stark, H., et al., A three-dimensional musculoskeletal model of the dog. Scientific reports, 2021. 11(1): p. $1-13$.

45. Favier, C., et al., An open-source musculoskeletal model of the lumbar spine and lower limbs: a validation for movements of the lumbar spine. Computer Methods in Biomechanics and Biomedical Engineering, 2021: p. 1-16.

46. Trinler, U., et al., Estimation of muscle activation during different walking speeds with two mathematical approaches compared to surface EMG. Gait \& posture, 2018. 64: p. 266-273.

47. Sukadarin, E.H., et al., 8 WRMP AND EVALUATION OF ERGONOMICS ASSESSMENT METHOD AMONG HARVESTERS OF OIL PALM PLANTATION. Occupational Safety and Health in Commodity Agriculture: Case Studies from Malaysian Agricultural Perspective, 2014: p. 275.

48. Syuaib, M.F., Ergonomic study on the manual harvesting tasks of oil-palm plantation in Indonesia based on anthropometric, postures and work motions analyses. Agricultural Engineering 
International: CIGR Journal, 2015. 17(3).

49. Bortoletto, R., E. Pagello, and D. Piovesan. Effects of reserve actuators on optimization solutions: From muscle force to joint stiffness. in 2015 IEEE International Conference on Rehabilitation Robotics (ICORR). 2015. IEEE.

50. Ng, Y.G., et al., Ergonomics observation: Harvesting tasks at oil palm plantation. Journal of occupational health, 2013: p. 13-0017-FS.

51. Hall, S.J., Basic biomechanics. 2015: McGraw-Hill Education.

52. Jelani, A.R., et al., Force and energy requirements for cutting oil palm frond. Journal of Oil Palm Research, 1998. 10(2): p. 10-24.

53. Michaud, F., et al., A fair and EMG-validated comparison of recruitment criteria, musculotendon models and muscle coordination strategies, for the inverse-dynamics based optimization of muscle forces during gait. Journal of NeuroEngineering and Rehabilitation, 2021. 18(1): p. 1-15.

54. Ma, Y., et al., The effect of musculoskeletal model scaling methods on ankle joint kinematics and muscle force prediction during gait for children with cerebral palsy and equinus gait. Computers in Biology and Medicine, 2021. 134: p. 104436.

\section{Figures}

\section{Figure 1}

Location of (a) IMU sensors on the joints of interest of a harvester, (b) EMG electrodes on muscles of interest of a harvester from posterior view and (c) right side view, with reference electrode at the C7 region.

\section{Figure 2}

A sequence of the harvesting motion of a real harvester during harvesting. 

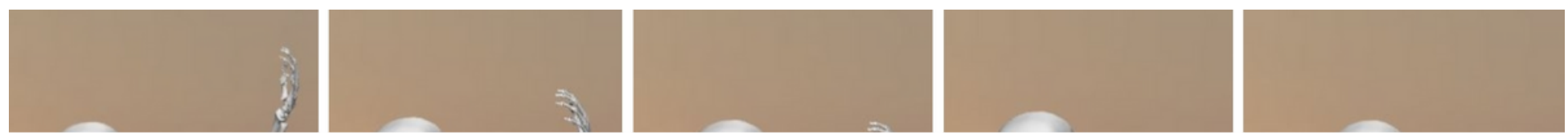

Figure 3

A sequence of the simulated harvesting motion of a harvester.

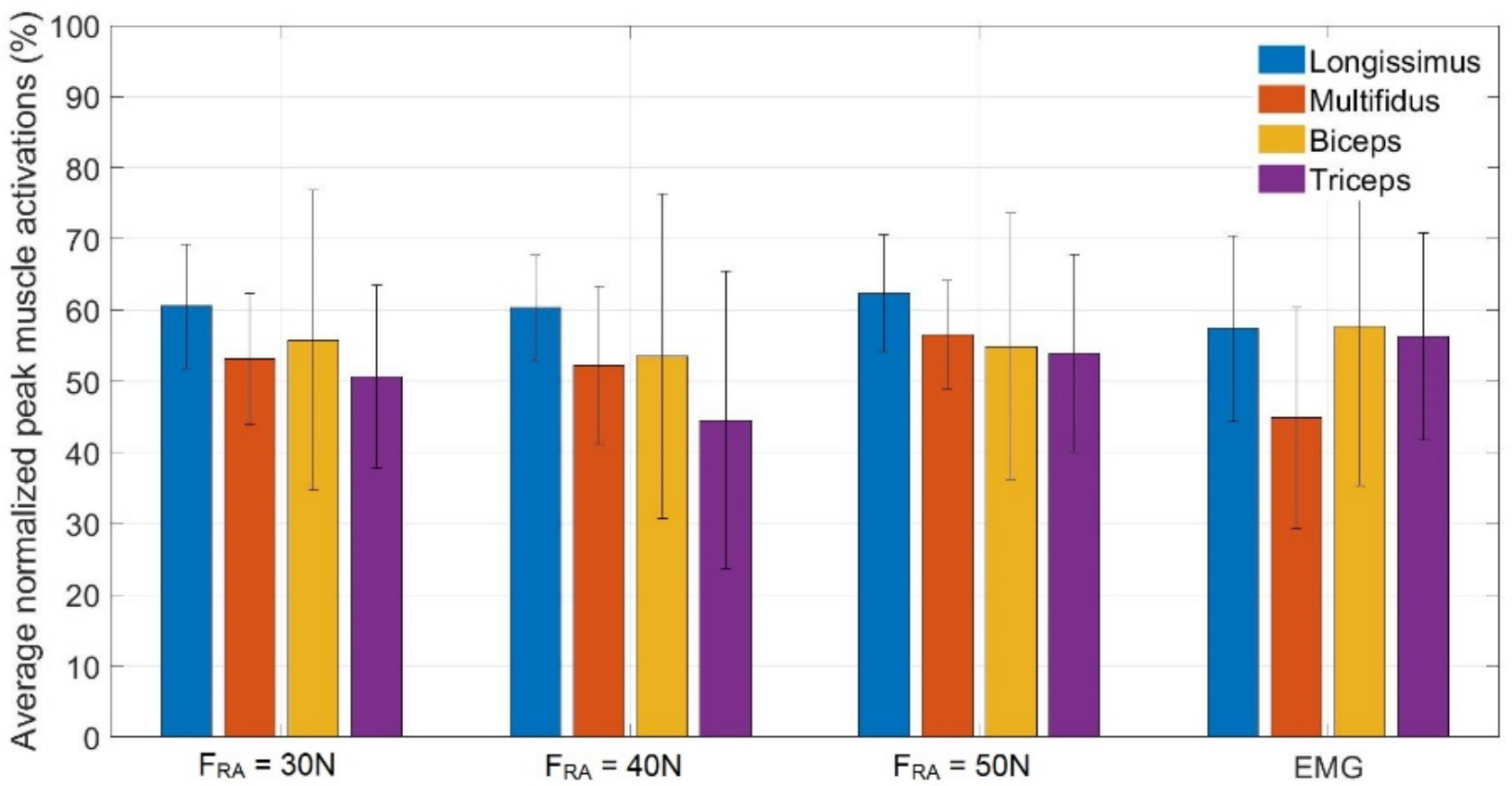

Figure 4

Average normalized peak muscle activations of harvesters in percentage $(n=6)$ during harvesting. 